\title{
Mobilité résidentielle, mobilité voiture et logiques familiales en Belgique : entre étalement de la périurbanisation et intra-urbanisation
}

\author{
Jean-Marie Halleux ${ }^{1}$
}

\begin{abstract}
Résumé - - De nombreux travaux montrent que les évolutions urbaines contemporaines s'inscrivent dans un processus de recomposition des villes induit par la mobilité facilitée et par la banalisation de la conduite automobile. Nous traitons cette thématique en étudiant, pour le contexte belge, comment le relâchement des freins à la mobilité interfère avec les logiques résidentielles des familles en formation. Un bilan sur la situation contemporaine est d'abord dressé. Nous y présentons les principaux liens entre choix résidentiels, classes d'âges et parcours de vie. Nous y analysons aussi les relations entre, d'une part, le constat de l'étalement périurbain et, d'autre part, la pression foncière à laquelle les ménages en formation ont à faire face. En seconde partie, la situation contemporaine est confrontée aux évolutions des cinq dernières décennies. Ces analyses réalisées pour la région urbaine de Liège permettent de combiner les trois grilles explicatives du moment du cycle de vie, de l'effet du renouvellement des générations et de l'impact du contexte socioéconomique. De ce travail, nous relevons que la mobilité facilitée conduit à dilater les inscriptions démographiques. Comme les groupes sociaux, les groupes démographiques sont, en effet, de plus en plus spatialement ségrégés dans une région urbaine très largement recomposée par la mobilité automobile.
\end{abstract}

Mots-clés - Étalement, Famille, Mobilité, Périurbanisation, Ville

\begin{abstract}
Residential mobility, car mobility and families' logics in Belgium: between periurbanisation sprawl and intra-urbanisation. - Numerous researches show that contemporary urban changes take place within a general transformation driven by increasingly easy travel and car mobility generalisation. This paper tackles this issue for the Belgian context. It analyses the relationships between, on the one hand, release of mobility constraints and, on the other hand, residential logics of young families. It first reviews the contemporary situation with a presentation of the major relationships between life cycle, age structure and residential choices. The current major links between peri-urbanisation sprawl and the land price pressure that young families have to deal with are also taken into account. In the second part, contemporary circumstances are compared with the situation during the last five decades. The analyses, which are realised for the Liège urban region, combine three explanatory dimensions: life cycle, generation replacement movement and socio-economic context. This leads to the conclusion that released mobility distends demographic settlements. Like social groups, demographic groups are, indeed, more and more spatially segregated within an urban region largely recomposed by car mobility.
\end{abstract}

Keywords - City, Family, Mobility, Peri-urbanisation, Spraw

1. Géographie économique/SEGEFA - LEPUR, Université de Liège, Allée du 6-Août n² 2, Bât. 11, 4000 Liège (Belgique). Tél : 32.(0)4.366.53.27 - Fax: 32.(0)4.366.56.30. Email : Jean-Marie.Halleux@ulg.ac.be 
Les conséquences clés de la facilité accrue aux déplacements sur les organisations urbaines sont désormais identifiées et nous savons aujourd'hui relier en système la dynamique des villes à la vitesse des déplacements (F. Beaucire, 2001). De nombreux travaux relèvent ainsi que les évolutions contemporaines s'inscrivent dans un processus de recomposition des systèmes et tissus urbains induit par le relâchement des contraintes de mobilité, principalement en raison de la banalisation de la conduite automobile ${ }^{2}$. Sur la base de ce constat, M. Wiel (1999a; 1999b) a proposé de désigner la transition urbaine comme étant, sur le long terme, le processus structurel de redéploiement des villes sous l'impact de l'auto-mobilité motorisée: "la transition urbaine serait le redéploiement - à potentiel identique d'interaction - des composants urbains sous l'effet d'une évolution du coût généralisé de déplacement, donc des arbitrages des ménages et des entreprises dans leur localisation et/ou leur tendance à la concentration" (M. Wiel, 1999a, p. 56).

Dans le cadre de cet article, nous proposons d'approfondir cette thématique en étudiant comment le relâchement des freins techniques et financiers à la mobilité interfère avec les logiques résidentielles des familles en formation. Pour répondre à ce questionnement, un bilan sur la situation contemporaine sera d'abord dressé. Nous y présenterons les principaux liens entre choix résidentiels, classes d'âges et parcours de vie. Nous y développerons également la relation entre la logique familiale de l'élargissement du ménage, l'étalement de la périurbanisation et la pression foncière. Dans une seconde partie, la situation contemporaine sera confrontée aux évolutions des cinq dernières décennies, ce qui permettra de combiner les trois grilles explicatives du moment du cycle de vie, de l'effet du renouvellement des générations et de l'impact du contexte socio-économique. Nous y verrons notamment comment la nouvelle norme d'emploi flexible accroît l'intra-urbanisation des jeunes ménages.

\section{LES CYCLES DE VIE, LES LOGIQUES RÉSIDENTIELLES ET L'ÉTALEMENT DE LA PÉRIURBANISATION: BILAN DE LA SITUATION CONTEMPORAINE}

\subsection{Les classes d'âges, les cycles de vie et les logiques résidentielles}

Construit à partir de données du Registre national, rassemblées, présentées et commentées par S. Savenberg et E. Van Hecke (1998), le graphique 1 identifie, pour l'ensemble de la Belgique et pour la période 1988-1996, le solde migratoire en fonction des classes d'âges des migrants et en fonction du degré d'urbanisation. Le degré d'urbanisation est basé sur une typologie concentrique des 589 communes belges (H. Van der Haegen et al., 1996), typologie prenant en compte les 17 régions urbaines du Royaume en différenciant cinq groupes annulaires: traditionnels;

- les villes centrales, soit les municipalités correspondant aux centres-villes

2. Voir par exemple: P.W.G. Newman et J.-R. Kenworthy, 1989; G. Dupuy, 1995; F. Ascher, 1995; G. Dubois-Taine et Y. Chalas, 1997; M. Wiel, 1999a et 1999b; J.-M. Halleux, 2001 et 2002. 


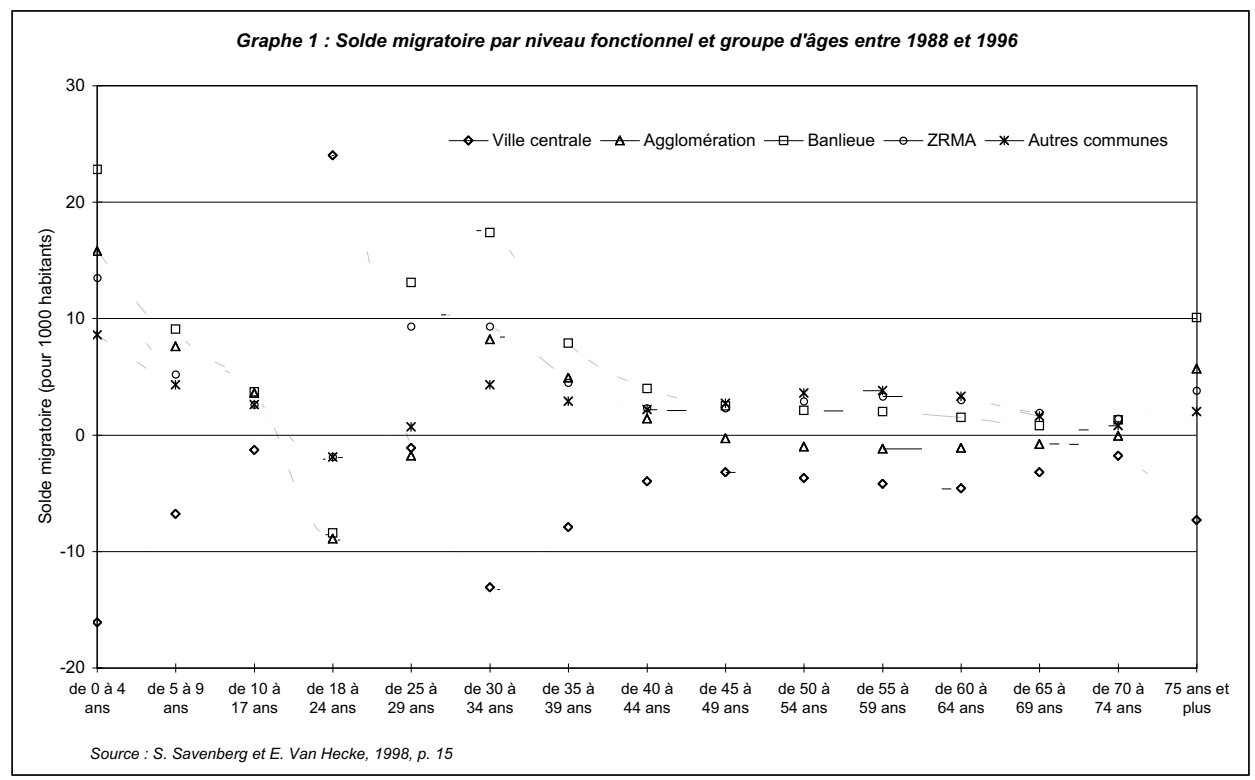

- les communes d'agglomérations, c'est-à-dire les entités marquées par une urbanisation morphologique continue depuis la ville centrale;

- la banlieue périurbaine, formée des communes dont la croissance démographique des dernières décennies a été alimentée par le déversement en provenance de l'agglomération et de la ville centrale;

- la zone résidentielle des migrants alternants, rattachée à la région urbaine en raison d'un fort développement de navettes pendulaires autochtones vers l'agglomération et la ville centrale;

- la catégorie autres communes, formée des 214 municipalités situées audelà des zones résidentielles des migrants alternants.

La prise en compte croisée de l'âge des migrants et du degré d'urbanisation des communes conduit à l'identification de trois mécanismes migratoires: les migrations centripètes des jeunes adultes, les migrations liées à l'agrandissement du ménage et les migrations des personnes âgées.

\section{L'intra-urbanisation des jeunes adultes}

On observe que les adultes de 18 à 24 ans forment le seul groupe pour lequel le solde migratoire des villes centrales est positif. On peut, à propos de ce mouvement centripète, utiliser le vocable général d'intra-urbanisation, les lieux d'arrivée étant, par rapport aux lieux de départ, plus proches des noyaux urbains (J.-M. Halleux, 2001, p. 72). Les intra-urbanisations des jeunes adultes, qui peuvent être considérées comme des "migrations d'émancipation" (J.-P. Grimmeau et al., 1998), marquent les espaces situés près des centres-villes. Généralement proches 
des institutions d'enseignement supérieur, ces quartiers disposent notamment d'une offre locative abondante. Nonobstant la motorisation banalisée, les centres-villes demeurent donc toujours attractifs pour le segment démographique des jeunes adultes, nous y reviendrons.

\section{L'agrandissement du ménage et le mouvement d'exurbanisation-périurbanisation}

À la suite de l'intra-urbanisation du groupe 18-24 ans, les autres mouvements migratoires sont tous centrifuges. Cela débute avec les migrations liées à l'agrandissement du ménage. C'est dans ce cadre que se déroule l'essentiel des processus d'exurbanisation et de périurbanisation, que nous définirons ici comme, d'une part, le départ des villes centrales et, d'autre part, le peuplement de la banlieue périurbaine et de la zone résidentielle des migrants alternants. Ces couronnes externes ne bénéficiant généralement que d'une accessibilité médiocre par les alternatives à la voiture, les développements qui y prennent place sont très largement dépendants de l'automobile. Avec le graphique 1, on vérifie que la périurbanisation est alimentée par les groupes d'âges compris entre 25 et 39 ans. On y observe également le mouvement des enfants quittant les villes avec leurs parents. C'est d'ailleurs pour le groupe des 0-4ans que l'exode urbain est le plus intense. Tout cela confirme le stéréotype du jeune couple avec enfants à la recherche d'un cadre périphérique mieux adapté à la vie familiale d'un ménage agrandi. Le mouvement d'exurbanisation-périurbanisation peut aussi être associé à l'idée d'une "ville-tremplin", que l'on quitte à la suite d'une stabilisation personnelle et/ou professionnelle.

Même si ce sont les banlieues périurbaines qui accueillent le plus de migrants durant la période d'agrandissement du ménage, les flux sont également conséquents vers l'agglomération et vers la zone résidentielle des migrants alternants. On observe en fait que les trentenaires optent plutôt pour les deux couronnes de l'agglomération et de la banlieue périurbaine, alors que les 25-29 ans sont proportionnellement plus nombreux à s'éloigner pour peupler la zone résidentielle des migrants alternants. Puisqu'une migration d'agrandissement du ménage est généralement synonyme d'acquisition du logement, cet éloignement est à relier aux mécanismes de l'immobilier résidentiel. Pour ces jeunes ménages, globalement peu fortunés, la volonté d'accéder à la propriété d'une maison unifamiliale oblige, désormais, à dépasser une première couronne devenue inabordable. En d'autres termes, les mécanismes de l'immobilier résidentiel engendrent un étalement de la périurbanisation, nous y reviendrons également.

Par rapport à la globalité des flux résidentiels, les migrations d'agrandissement du ménage à caractère périurbain sont largement dominantes en Belgique. Différentes enquêtes ont donc été menées afin de préciser le profil et les motivations des migrants concernés (J.-M. Halleux, 1999; L. Brück et al., 2000). Comme nous venons de le voir, la décision effective de l'exurbanisation et de l'installation en périphérie est très généralement liée aux étapes du parcours de vie. Une autre conclusion importante des enquêtes menées sur les choix périurbains est qu'ils 
s'inscrivent dans une pensée collective très forte qui considère la "campagne" comme la localisation résidentielle idéale. Les personnes attirées par la campagne ne rejettent d'ailleurs pas nécessairement la "ville", mais cette dernière est considérée comme un lieu d'activité plutôt que comme un lieu de vie, surtout s'il s'agit d'élever des enfants. Lorsque le ménage quitte une localisation centrale pour s'établir en périphérie, la structure familiale est déjà formée et le lieu de départ est, en général, quitté par un couple. C'est l'insatisfaction par rapport aux caractéristiques du logement qui apparaît alors comme la cause première de l'exurbanisation. Le désir de quitter un intérieur trop petit, d'échanger un appartement pour une maison unifamiliale et l'absence de jardin sont des facteurs très souvent évoqués. Ils sont bien sûr en parfaite cohérence avec les besoins d'espaces intérieurs découlant d'événements comme le mariage ou la parenté. En Belgique, où l'acquisition immobilière est valorisée depuis plus d'un siècle, les motifs familiaux de l'exurbanisation sont parfaitement corrélés avec cet autre facteur-clé du désir de devenir propriétaire. À ce propos, la sociologie nous apprend que, pour le jeune ménage, l'accès à la propriété permet d'acquérir "une maison au sens de maisonnée, c'est-à-dire de créer un groupe social uni par les liens de l'alliance et de la parenté, que redoublent les liens de la cohabitation" (P. Bourdieu et al., 1990, p. 7).

\section{Les mobilités résidentielles des plus de cinquante ans}

Pour les tranches d'âges plus avancées, le solde migratoire des villes centrales demeure négatif. Contrairement à une idée reçue, les principales villes belges ne sont donc pas attractives pour les populations âgées: il n'est "jamais trop tard" pour s'exurbaniser. À partir de 50 ans, le solde migratoire devient également négatif pour les communes d'agglomération. Si les migrations résidentielles des personnes âgées de plus de 50 sont centrifuges et plus diffuses encore que les migrations d'agrandissement du ménage, cela s'explique par la disparition de la contrainte d'accessibilité à l'emploi. Cette disparition des contraintes de la mobilité pendulaire permet d'optimiser les deux autres facteurs de localisation du caractère attractif du lieu de vie et de la proximité du milieu d'origine (H. Van der Haegen et al., 2000). Au-delà de 75 ans, le solde migratoire devient de nouveau très négatif pour les villes centrales. Sans doute la dépendance vis-à-vis des enfants et la localisation des maisons d'accueil expliquent-elles cet autre type d'exode urbain?

\subsection{De l'acquisition de la maisonnée à l'étalement de la périurbanisation}

La transformation indirecte du gain de vitesse en périurbanisation est un fondement essentiel de l'évolution urbaine. Aujourd'hui, c'est bien parce que les familles peuvent se déplacer plus vite qu'elle peuvent localiser la "maisonnée" sur des territoires élargis. Afin d'étudier ce mouvement massif de périurbanisation, il est utile de différencier le desserrement de l'étalement (V. Fouchier, 2001). Nous regroupons sous le premier vocable l'augmentation de la surface résidentielle par personne. Les familles choisissant les périphéries pour disposer de logements 
vastes et confortables, il s'agit là d'une évolution positive. Par étalement, nous entendons la distanciation des lieux-fonctions. En termes de surcharges collectives, un étalement exagéré est problématique. Il entraîne, en effet, un accroissement inutile des déplacements et des trafics.

Par simplification géométrique, l'étalement peut se mesurer par rapport aux centres traditionnels. Une manière commode de l'appréhender consiste alors à calculer la distance moyenne entre un indicateur de l'urbanisation et le point focal du système urbain (V. Fouchier, 2001, p. 30). Le graphique 2 reprend ce type d'analyse pour la région fonctionnelle de Liège et pour les communes wallonnes du bassin d'emploi de la région fonctionnelle de Bruxelles ${ }^{3}$ : il porte sur l'évolution de la distance moyenne des immigrations communales au centre de ces deux villes ${ }^{4}$. Clairement, la tendance est à l'éloignement ou, en d'autres termes, à l'étalement. Le constat géométrique de l'étalement corrobore les travaux démographiques récents sur le peuplement des régions fonctionnelles, travaux qui identifient un tassement de la croissance des premières couronnes périurbaines et, à l'inverse, un déversement au sein des territoires les plus excentrés (T. Eggerick et C. Capron, 2001).

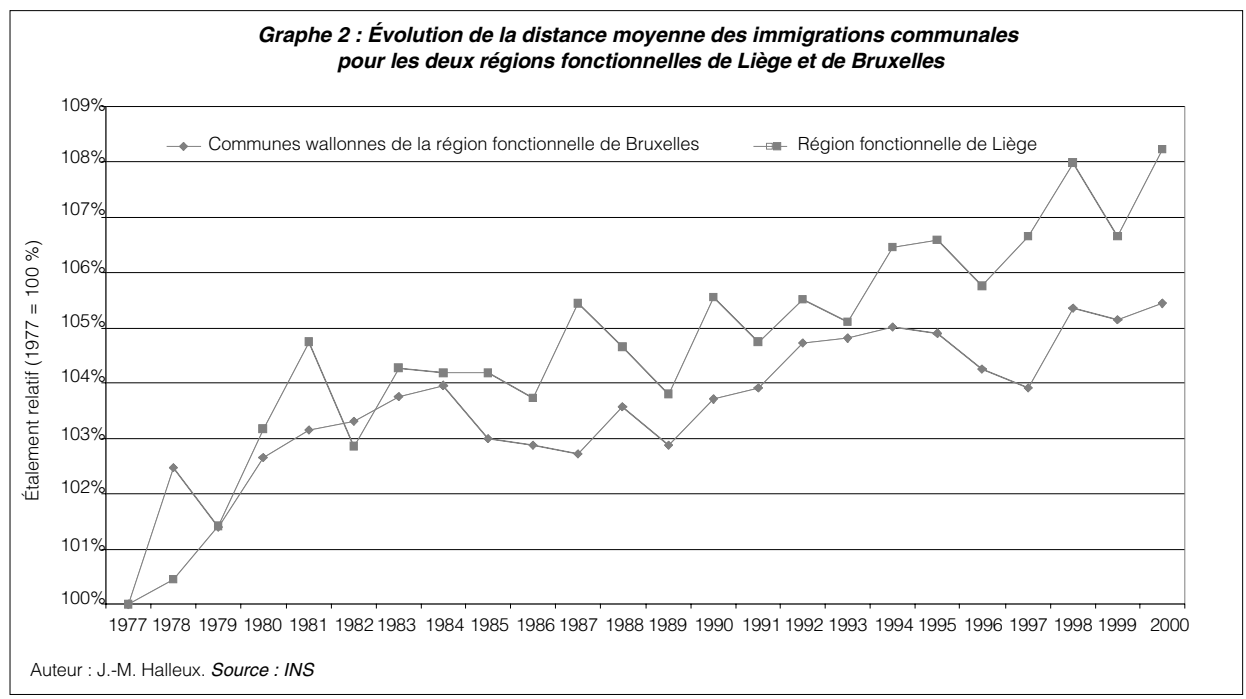

3. La délimitation des régions fonctionnelles est basée sur les relations quotidiennes entre lieu de résidence et lieu de travail. La cartographie et les détails méthodologiques ont été publiés par le Service des Études et de la Statistique (SES) de la Région wallonne (Y. De Wasseige et al., 2000).

4. Cette distance se calcule comme une moyenne des distances entre les différentes communes des régions fonctionnelles et la commune centrale, le calcul étant en fait pondéré par le volume de l'immigration communale. 


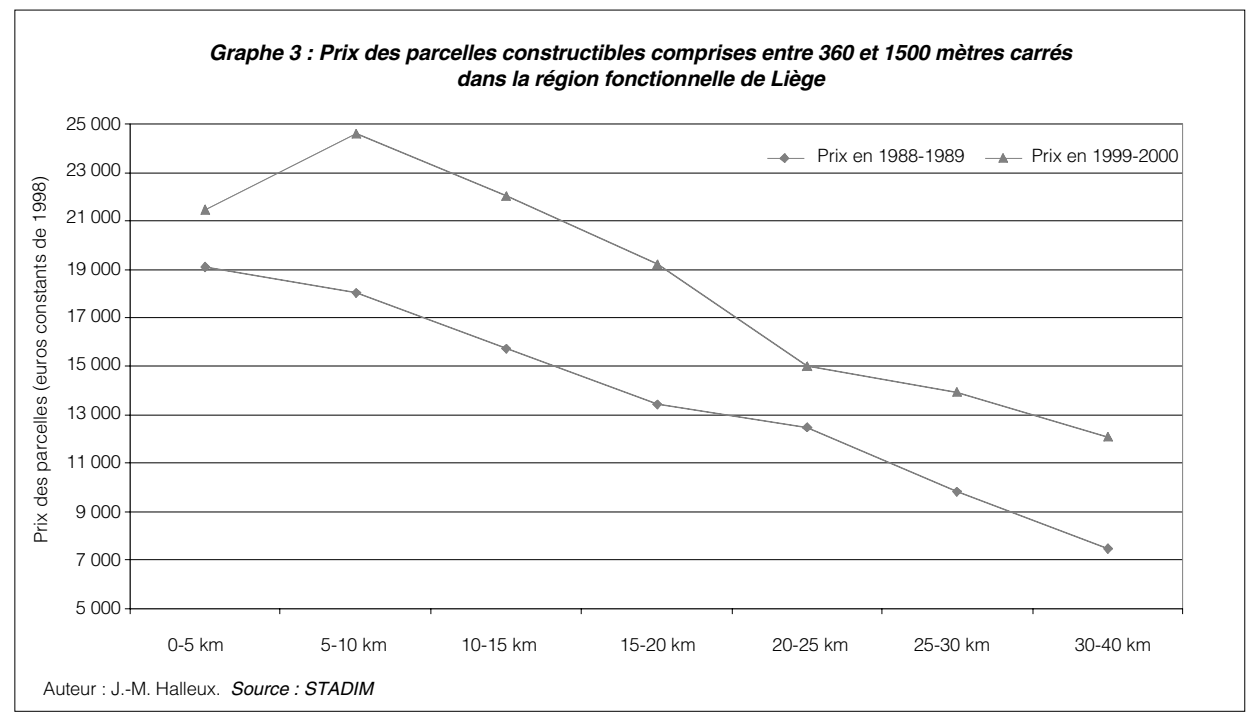

La tendance à l'étalement peut résulter de causes distinctes. Cela pourrait tout d'abord tenir à des raisons de capacité physique: l'urbanisation entraînant une consommation perpétuelle en ressources foncières, les nouveaux développements seraient rejetés en dehors d'agglomérations de plus en plus étendues. Cette hypothèse de l'étalement de capacité physique semble toutefois réfutée par les études portant sur la configuration des zones juridiquement urbanisables. En effet, les terrains vierges et constructibles abondent dans l'ensemble des communes situées en première couronne périphérique, à Liège comme à Bruxelles (Ministère de la Région wallonne, 2002). Un autre facteur d'étalement correspond à la rétention (étalement de rétention foncière). Certains terrains situés à proximité des noyaux urbains traditionnels n'étant pas disponibles sur le marché, cela éloigne les maîtres d'ouvrage (Dubois et al., 2002). Au-delà de ce mécanisme juridique, les logiques économiques des marchés participent également à l'étalement. À ce propos, nous avons déjà pu relever ci-dessus, concernant les lieux d'implantation des jeunes familles, que les ménages les moins nantis doivent désormais dépasser la banlieue périurbaine pour accéder à la propriété d'une maison individuelle. Cet étalement de pression foncière, qui générera ensuite des déplacements inutilement allongés, n'est pas dû à une absence d'offre physiquement accessible, ni même à une absence d'offre disponible sur le marché, mais bien aux prix pratiqués. Le prix des parcelles constructibles (graphe 3) ou des habitations étant décroissants en fonction de l'éloignement par rapport à la ville, c'est en effet pour permettre la rencontre entre les ressources financières de la demande et les prix d'offre qu'il faut s'éloigner. 


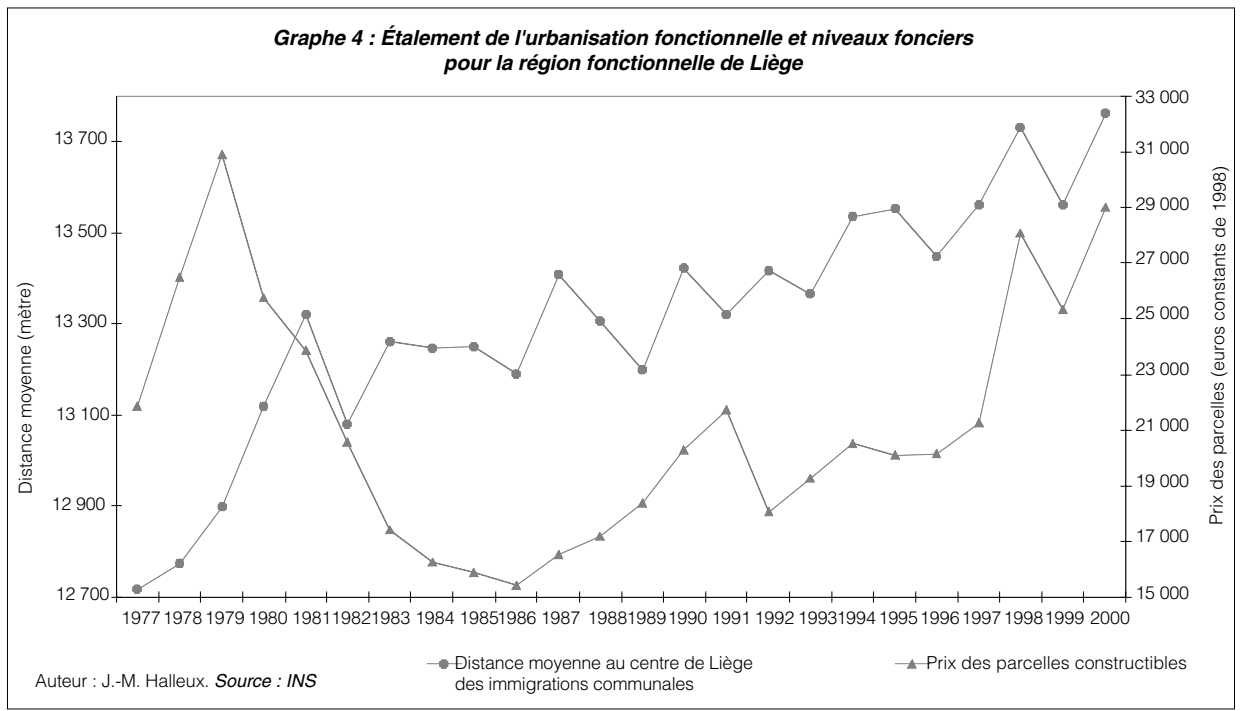

L'hypothèse de l'étalement de pression foncière est corroborée par les graphiques 4 et 5 . Sur base de ces deux graphiques évolutifs, nous vérifions, en effet, qu'une croissance des niveaux fonciers oblige les ménages à élargir leur zone de prospection foncière et à établir la maisonnée sur des sites de plus en plus éloignés des noyaux traditionnels.

Le graphique 4 porte sur la région fonctionnelle de Liège et rassemble les deux variables du prix des parcelles et de la distance moyenne au centre de Liège des immigrations communales 5 . Trois périodes peuvent y être distinguées. À la première période, comprise entre 1977 et 1979 pour les niveaux de prix et 1977 et 1981 pour l'étalement, correspond une étroite corrélation temporelle entre l'inflation foncière et l'éloignement. On est ici en présence de statistiques déterminées par les décisions d'achats et de localisations résidentielles antérieures à la crise du début des années 1980. Le cycle conjoncturel de la décennie 1980 se marque ensuite très clairement sur les niveaux fonciers. Entre 1981 et 1986, en réponse à la baisse des prix, on observe une stabilisation de l'étalement. Pour les ménages qui n'étaient pas directement affectés par la crise économique et par la montée du chômage, il devient alors plus aisé d'acquérir une parcelle en première couronne. Depuis la sortie de la crise, on assiste globalement à une hausse continue des niveaux fonciers moyens, dont le corollaire correspond à une poursuite de l'étalement.

Le graphique 5 portant sur les communes wallonnes de la région fonctionnelle de Bruxelles reprend la même variable du prix des parcelles constructibles, qui est ici confrontée à une mesure de l'étalement de la périurbanisation morphologique, c'est-à-dire la distance moyenne au centre de Bruxelles des demandes de permis

5. Il s'agit de la variable reprise au graphique 2, mais cette fois en valeurs absolues. 


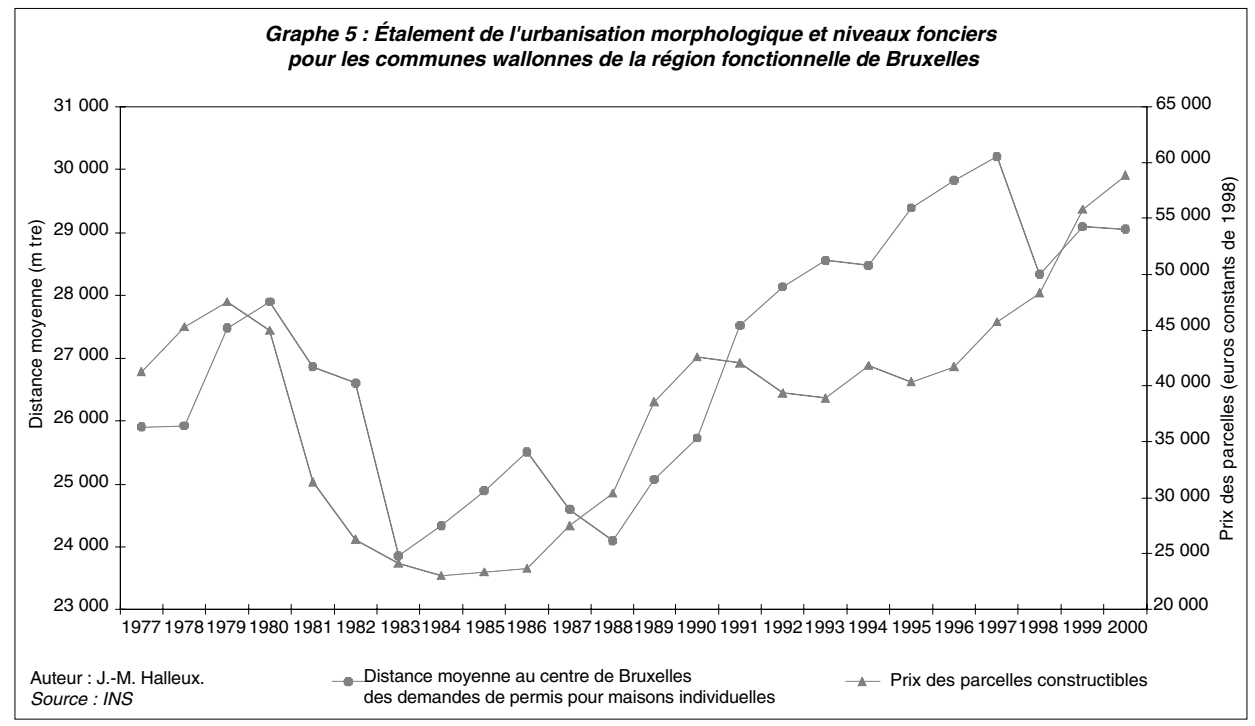

pour maisons individuelles. La corrélation temporelle entre les deux variables est ici plus nette qu'à Liège, ce qui tient tout d'abord à la méthode de construction des données. En effet, la zone bruxelloise étant plus vaste que la région fonctionnelle de Liège, les indicateurs y sont moins affectés par la variabilité statistique. La plus forte corrélation temporelle tient également aux différentiel foncier entre Liège et Bruxelles. Les niveaux de prix étant significativement plus élevés au sein des communes sous l'influence de la capitale, la contrainte foncière y est plus forte.

\section{LES MOBILITÉS RÉSIDENTIELLES DES FAMILLES DANS UNE PERSPECTIVE TEMPORELLE}

Après avoir construit un bilan sur les articulations contemporaines entre la banalisation de la conduite automobile et les logiques développées par les familles pour leurs choix de localisation, nous proposons maintenant d'approfondir le sujet par une mise en perspective temporelle. Notre objectif est d'étudier comment les relations entre les choix résidentiels et les cycles de vie ont évolué au cours des cinq dernières décennies. Cet approfondissement des travaux réalisés par S. Savenberg et E. Van Hecke (1998) nous permettra d'enrichir la vision contemporaine par les deux grilles d'analyse du renouvellement des générations et des mutations du contexte socio-économique. Complémentaire à la grille du cycle de vie, qui ne relie les décisions et les besoins des individus qu'à leur âge, la prise en compte de l'inertie du renouvellement des cohortes démographiques permettra de vérifier comment le simple remplacement des anciennes générations par des cohortes plus motorisées participe à l'accroissement de la mobilité voiture. Pour compléter le 
recadrage temporel, nous intégrerons également le contexte socio-économique en reliant la nouvelle norme d'emploi flexible à l'intensification de l'intra-urbanisation des jeunes ménages.

\subsection{Présentation de la zone d'étude et de la méthodologie générale}

C'est l'arrondissement de Liège que nous avons choisi comme zone d'étude afin d'établir la mise en perspective temporelle des relations entre la mobilité voiture et les mobilités résidentielles des familles, cela pour la période 1948-2000 et sur base d'un indicateur des transferts démographiques: le taux d'échanges intraarrondissement.

Cet indicateur rend compte des flux migratoires entre trois couronnes concentriques. La première couronne, que nous avons dénommée la ville centrale, correspond au territoire de la Ville de Liège antérieur à la fusion des communes de 1977. La seconde couronne, dénommée la couronne suburbaine, reprend, hors ville centrale, les entités englobées au sein de l'agglomération de 1970 (M. Pattyn et H. Van der Haegen, 1979). Il s'agit là de communes dont l'urbanisation, généralement associée au développement industriel et antérieure à la Seconde Guerre Mondiale, s'est intimement articulée aux réseaux de transports en commun. La troisième couronne, dénommée périurbaine, est formée des autres communes de l'arrondissement. La densité y est relativement faible et l'accessibilité par les modes alternatifs à la voiture souvent médiocre.

Lors du dernier demi-siècle, l'arrondissement de Liège a été marqué par un fort mouvement d'exurbanisation-périurbanisation. Pour la ville centrale, la population est ainsi passée de 156208 habitants en 1948 à 103161 habitants en 2000. À l'inverse, la couronne périurbaine a connu une intense croissance. Sur la même période, son volume de population progresse de 132265 à 210660 habitants. Pour la couronne suburbaine, on observe un léger tassement, de 275891 habitants en 1948 à 271857 habitants en 2000.

La variable du taux d'échanges intra-arrondissement s'appuie sur l'utilisation des statistiques démographiques par tranches d'âges. En raison de la disponibilité de cette donnée lors des recensements, nous avons pu en disposer pour 1948, 1962, 1971, 1981, 1991 et $2000^{6}$. La première étape de la construction du taux d'échanges intra-arrondissement a consisté, pour chaque tranche d'âges et pour chaque période comprise entre deux années d'observation, dans le calcul d'un ratio de présence intra-arrondissement. Par exemple, pour un volume démographique de 10000 pour la cohorte 50 ans en 1981 et de 8000 pour la cohorte 60 ans en 1991, le ratio de présence intra-arrondissement afférent est de $80 \%$. Ce ratio intègre à la fois l'impact de la mortalité (le ratio est faible pour les tranches d'âges

6. Les résultats de l'Enquête socio-économique de 2001 n'étant pas disponibles lors des traitements, ce sont les données relatives à la situation au $1^{\text {er }}$ janvier 2000 qui ont été utilisées. À la différence des autres données, la source originelle correspond ici au Registre National. 
où la mortalité est forte) et l'effet global des migrations avec l'extérieur de l'arrondissement. Disposant de ce ratio pour chaque cohorte et pour chaque période, il est possible d'établir, pour les trois couronnes, une population théorique attendue, représentant le volume d'une population ayant évolué comme l'ensemble de l'arrondissement. En tablant sur un différentiel négligeable entre les trois couronnes quant à la mortalité et quant aux migrations extra-arrondissement, il devient ensuite possible de quantifier les échanges au sein de l'arrondissement. Pour cela, il faut simplement comparer la population réelle à la population théorique attendue. Nous avons choisi de le faire en construisant un taux d'échanges intra-arrondissement, qui se calcule de la manière suivante:

Taux d'échanges intra-arrondissement $=\underline{\text { Population réelle }- \text { Population attendue }}$ Population attendue

Le tableau 1 rassemble la statistique du taux d'échanges intra-arrondissement. La valeur de $-3 \%$ relative à la ville centrale pour la période 1948-1962 signifie que la population réelle en 1962 représente $\pm 97 \%$ de la population théorique calculée en appliquant le taux de présence intra-arrondissement à la structure par âge de 1948. Le tableau 1 permet déjà de dresser un bref historique de l'exurbanisation et de la périurbanisation. On voit que si ces deux tendances caractérisent l'ensemble des périodes, le paroxysme est clairement atteint lors de la décennie 1970. Pour la

\begin{tabular}{|l|c|c|c|}
\hline \multicolumn{3}{|c|}{ Tableau 1: Taux d'échanges intra-arrondissement } \\
\hline Période & Ville centrale & Couronne suburbaine & Couronne périurbaine \\
\hline $1948-1962$ & $-3 \%$ & $0 \%$ & $2 \%$ \\
\hline $1962-1971$ & $-3 \%$ & $-1 \%$ & $6 \%$ \\
\hline $1971-1981$ & $-9 \%$ & $-2 \%$ & $11 \%$ \\
\hline $1981-1991$ & $-3 \%$ & $-3 \%$ & $7 \%$ \\
\hline $1991-2000$ & $-2 \%$ & $-1 \%$ & $3 \%$ \\
\hline
\end{tabular}

période 1971-1981, le taux d'échanges relatif à la ville centrale est de -9\%, ce qui implique un départ net de $9 \%$ des urbains liégeois vers les deux autres couronnes. Pour la même période, la valeur relative à la couronne périurbaine est de $+11 \%$, rendant par-là compte d'une arrivée intra-arrondissement représentant $11 \%$ de la population théorique attendue.

\section{2. Évolution des taux d'échanges intra-arrondissement pour la ville centrale et la couronne périurbaine: le poids du contexte socio-économique global}

Le taux d'échanges intra-arrondissement peut être calculé pour chaque âge. Avec les graphes 6 et 7, c'est le résultat d'une agrégation par groupe quinquennal 


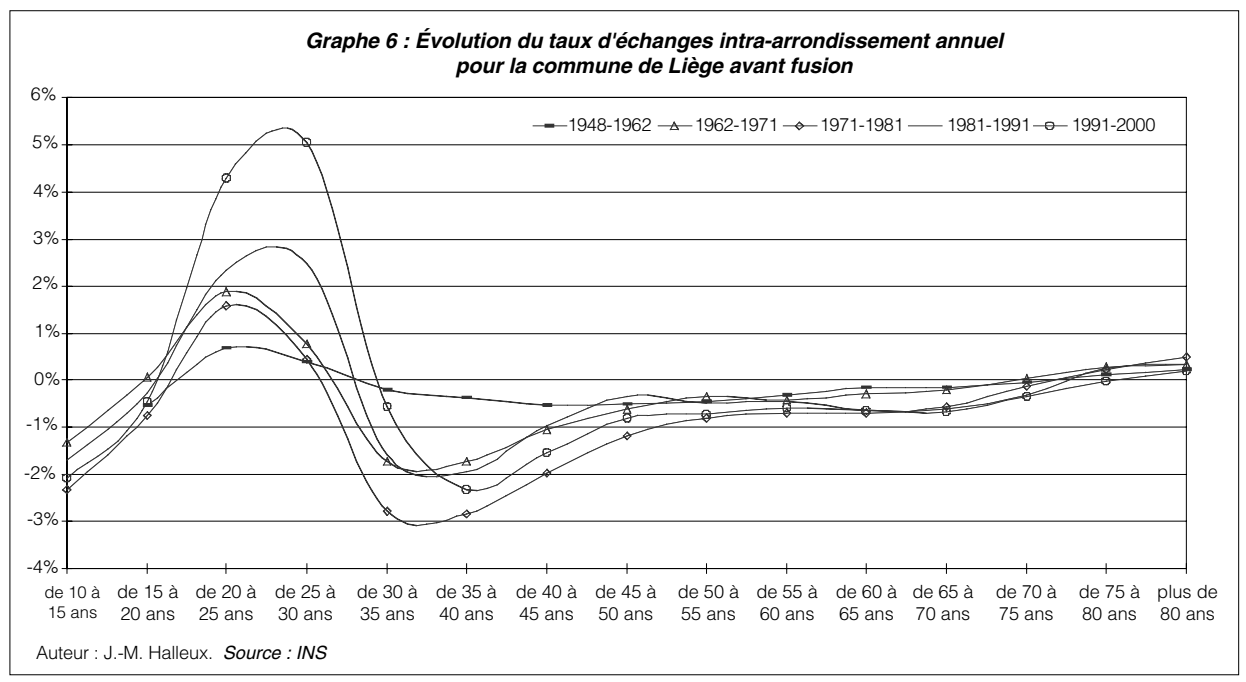

qui est présenté. Le taux d'échanges peut aussi être calculé pour les différentes périodes comprises entre les années pour lesquelles les structures par âges ont été publiées. Pour les graphes 6 et 7 , ce sont les cinq périodes successives comprises entre chacune des six années de disponibilité qui sont étudiées, cela pour la ville centrale et pour la couronne périurbaine. À propos de ces deux graphes, signalons encore que les valeurs ont été relativisées par le nombre d'années comprises entre les périodes d'observation (calcul d'un taux annuel) ${ }^{7}$ et que le premier groupe d'âge complet correspond à la classe des 10-15 ans ${ }^{8}$.

Pour la ville centrale comme pour la couronne périurbaine, les graphiques 6 et 7 vérifient l'allure générale des courbes présentées ci-dessus sur base des données du solde migratoire (graphique 1). Pour l'ensemble des périodes prises en compte, on y retrouve à la fois l'intra-urbanisation des jeunes adultes dans la vingtaine et l'exurbanisation-périurbanisation des trentenaires'.

Bien que présents dès la période 1948-1962, les trois processus d'intraurbanisation, d'exurbanisation et de périurbanisation n'étaient alors que faiblement

7. Mode de calcul de la variable présentée dans les graphes 6 et 7 :

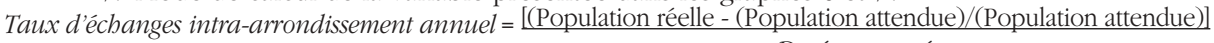
Durée en années

8. Le calcul de la population théorique étant basé sur la structure par âge en début de période, la simulation ne permet pas d'intégrer les individus n'étant pas encore nés à cette époque. Pour la courbe relative à la période 1948-1962, le premier groupe quinquennal complet correspond ici à la classe des 15-20 ans, ce qui tient bien sûr à la plus longue durée entre les deux époques d'observation.

9. Si un décalage d'approximativement 5 années est observé entre le graphique 1 et les graphiques 6 et 7, cela résulte simplement d'un différentiel méthodologique quant au moment où l'âge est pris en compte. Alors que la figure 1 porte sur l'âge au moment de la migration, la construction méthodologique des graphes 6 et 7 implique de le considérer à la fin de la période d'analyse. 


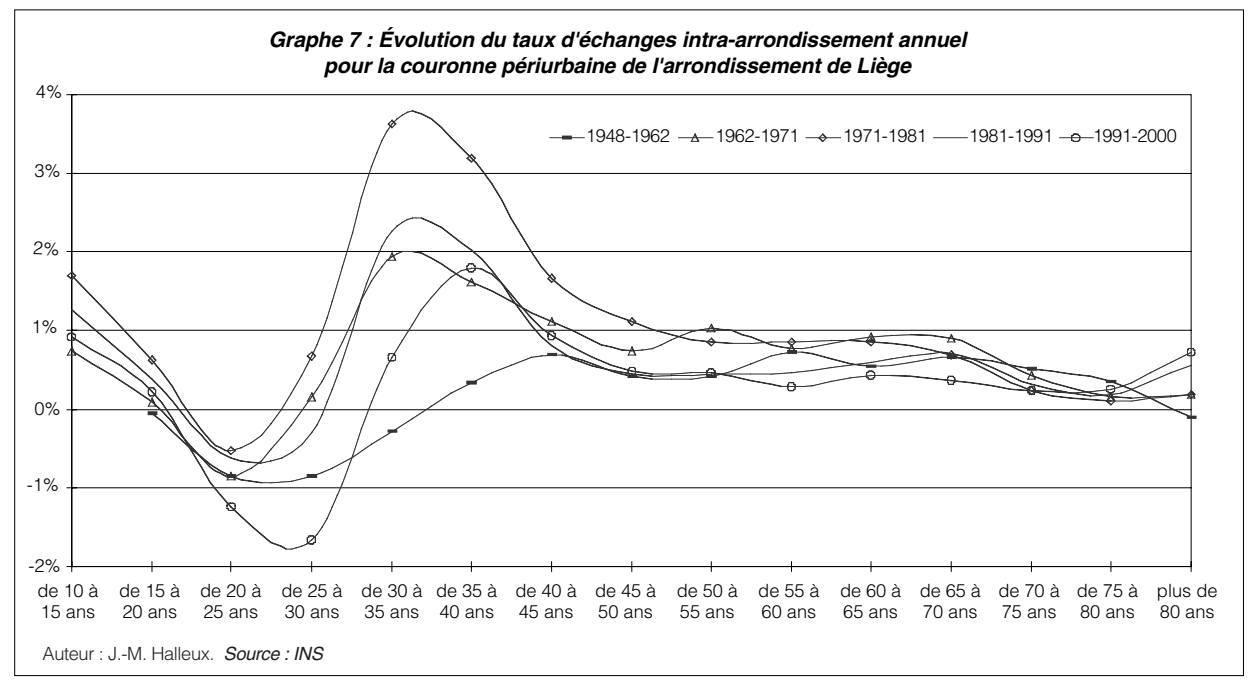

marqués, représentant une valeur de taux d'échanges annuel inférieur au pourcent. Par la suite, on assiste à leur accentuation. S'il est désormais bien connu que la vitesse des déplacements conduit à accroître la ségrégation spatiale par la spécialisation sociale et fonctionnelle de zones entières à l'intérieur de vastes régions urbaines (F. Ascher, 2000, p. 56; M.-L. De Keersmaecker, 2002), il ressort des processus évolutifs étudiés que la dilatation des territoires de la quotidienneté urbaine provoque également une dilatation des inscriptions démographiques. Les proximités résidentielles intragénérationnelles sont ainsi de plus en plus fortes dans la mosaïque territoriale en émergence. Efforçons nous maintenant d'examiner les processus qui expliquent cette situation.

\section{Le duo exurbanisation-périurbanisation}

Avec les graphiques 6 et 7, on vérifie que le double mouvement de sortie de la ville et d'installation en couronne périurbaine s'accentue dès les années 1970, période pendant laquelle il présente la même intensité que lors de la décennie 1980. Sur le plan quantitatif, voilà donc quatre décennies que la motorisation banalisée dilate l'espace-temps en intégrant les franges périphériques aux systèmes de la quotidienneté urbaine. Lors des décennies 1960 et 1980, on observe, pour les deux groupes 3035 ans et 35-40 ans, des taux moyens annuels proches de - $2 \%$ pour la ville centrale et de $+2 \%$ pour la couronne périurbaine. Lors de ces deux décennies, l'exurbanisation et la périurbanisation sont donc le fait d'environ $20 \%$ des trentenaires.

C'est ensuite lors de la décennie 1970 que se développent les flux les plus massifs. Pour les groupes 30-35 ans et 35-40 ans, on est ici en présence de taux annuels proches de $-3 \%$ pour la ville centrale et d'environ $+3,5 \%$ pour la couronne périurbaine. L'exurbanisation et la périurbanisation sont cette fois le fait du tiers des trentenaires. Faisant suite à plus de vingt années de croissance économique intense, 
nous sommes alors au paroxysme de la phase fordiste de développement et d'organisation sociale basée sur la régulation de l'État-Providence et sur la croissance du pouvoir d'achat des travailleurs-consommateurs. Il en résulte une amélioration considérable des infrastructures, une motorisation désormais largement accessible, une confiance des familles dans leur avenir économique et, en conséquence directe, un potentiel important pour la périurbanisation.

Avec la crise économique de la décennie 1980, on observe un reflux. Lors de la décennie 1990, si le taux de sortie est moindre chez les 30-35 ans, il est par contre très élevé chez les 35-40 ans. Par rapport à la période précédente, la ville est quittée plus tard, mais elle est toujours, in fine, autant quittée.

\section{L'intra-urbanisation des jeunes adultes}

Pour l'intra-urbanisation des jeunes adultes, on observe un renforcement continu de la tendance, renforcement particulièrement affirmé lors de la décennie 1990, période pendant laquelle le taux d'échanges annuel pour les 25-30 ans dépasse les $+5 \%$ pour la ville centrale. Cela signifie que le ratio entre la population réelle au $1^{\mathrm{er}}$ janvier 2000 et la population théorique, telle qu'on peut l'attendre sur base du volume des 16-21 ans en 1991, est d'approximativement $150 \%$. C'est donc au moins un tiers des liégeois appartenant au groupe des 25-30 ans en 2000 qui n'étaient pas présents sur le territoire de la ville centrale en 1991. Nous pouvons déduire de ces chiffres que l'attractivité croissante des tissus urbains centraux sur les jeunes adultes permet d'expliquer la moindre dépopulation constatée à Liège lors des dernières années. Sans doute ce facteur explicatif partiel est-il d'ailleurs également valable pour rendre compte de l'amélioration récente des bilans démographiques enregistrés pour d'autres grandes villes belges, notamment Bruxelles et Gand?

Parallèlement à l'accentuation des mouvements d'entrées et de sorties de ville, les graphes 6 et 7 attestent aussi d'un allongement de la durée des étapes du parcours de vie. Depuis les années 1980, l'intra-urbanisation apparait de plus en plus tardive. Elle était plutôt le fait des 20-25 ans entre 1948 et 1981 et, aujourd'hui, elle est plus importante pour les 25-30 ans. De même, nous avons déjà relevé que l'exurbanisation est également retardée. Répartie sur l'ensemble de la trentaine entre 1961 et 1991, elle tend depuis lors à se concentrer à la fin de cette période.

Apporter une explication au renforcement de l'intra-urbanisation des jeunes adultes conduit à suivre la thèse de M. Van Criekingen (2001) quant à l'impact des restructurations post-fordistes sur l'émergence d'une génération de captifs transitoires et de familles juvéniles flexibles. Si la ville a toujours été un lieu d'accueil pour les populations jeunes en recherche d'interactions sociales et économiques, elle est sans doute, en raison des difficiles stabilisations professionnelles et personnelles contemporaines, une étape résidentielle de plus en plus obligée. "En effet, les contraintes pesant sur la stabilisation familiale et professionnelle des jeunes adultes se sont nettement accrues depuis trois décennies ${ }^{10}$. Elles ont favorisé la constitution de 
nombreux petits ménages formés de jeunes adultes, issus des classes moyennes pour la plupart et souvent qualifiés, qui occupent une position transitoire dans le cycle de vie (entre décohabitation parentale et fixation familiale) et sur le marché du travail (entre fin des études post-secondaires et stabilisation professionnelle). La segmentation du marché du logement urbain et des contraintes spécifiques de reproduction sociale transforment une part importante de ces ménages en "captifs transitoires" des espaces urbains anciens. Aux mêmes âges, les parents de ces jeunes adultes avaient un accès plus immédiat à la propriété, principalement dans les banlieues résidentielles, en relation avec des perspectives familiales et professionnelles établies plus rapidement” (M. Van Criekingen, 2001, p. 242).

\section{L'attractivité déclinante de la couronne périurbaine}

Pour la couronne périurbaine, la période 1991-2000 est marquée par une attractivité déclinante (tableau 1). Cela tient d'abord au ratio fortement négatif pour le groupe des 25-30 ans (près de - $2 \%$ ), ce qui peut s'expliquer par la réintégration urbaine de cohortes s'étant précédemment installées dans le périurbain avec leurs parents lors de la décennie 1970. Le faible bilan pour la démographie de la couronne périurbaine lors de la décennie 1990 est également lié à un faible ratio pour les trentenaires (moins de $1 \%$ pour le groupe 30-35 et moins de $2 \%$ pour le groupe 35-40). Ces valeurs apparaissant assez basses par rapport aux niveaux de l'exode depuis la ville centrale, on peut en supposer qu'une part importante de l'exurbanisation profite désormais à des espaces situés au-delà de l'arrondissement, confirmant par-là le constat de l'étalement. Parallèlement, le transfert de jeunes ménages originaires de la couronne périurbaine intra-arrondissement vers une seconde couronne plus large est également susceptible de rendre compte de l'attractivité déclinante de cet espace pour les 20-40 ans. En bref, l'acquisition de la maisonnée oblige, de plus en plus, à franchir les limites de l'arrondissement.

\subsection{Les volumes d'échanges par cohortes entre 1948 et 2000 : l'influence du renou- vellement des générations}

Les taux d'échanges repris dans les graphes 6 et 7 ont été calculés pour les cinq périodes successives comprises entre les six époques de disponibilité des données. Avec la figure 8, c'est la même variable qui a été construite, mais cette fois pour l'ensemble de la période 1948-2000 et pour les seuls groupes d'âges audelà de 30 ans, cela afin de construire une analyse de la temporalité longue. En

10. En Belgique, $13 \%$ des moins de 25 ans étaient employés sous contrat temporaire au début des années 1990. Cette proportion est passée à $25 \%$ pour le même groupe d'âge en 1997. Toujours en Belgique, on note une nette hausse depuis la fin des années 1970 de l'occurrence du retour à une période de chômage après un premier emploi, même parmi les jeunes diplômés de l'enseignement supérieur (M. Van Criekingen, 2001, p. 73). 


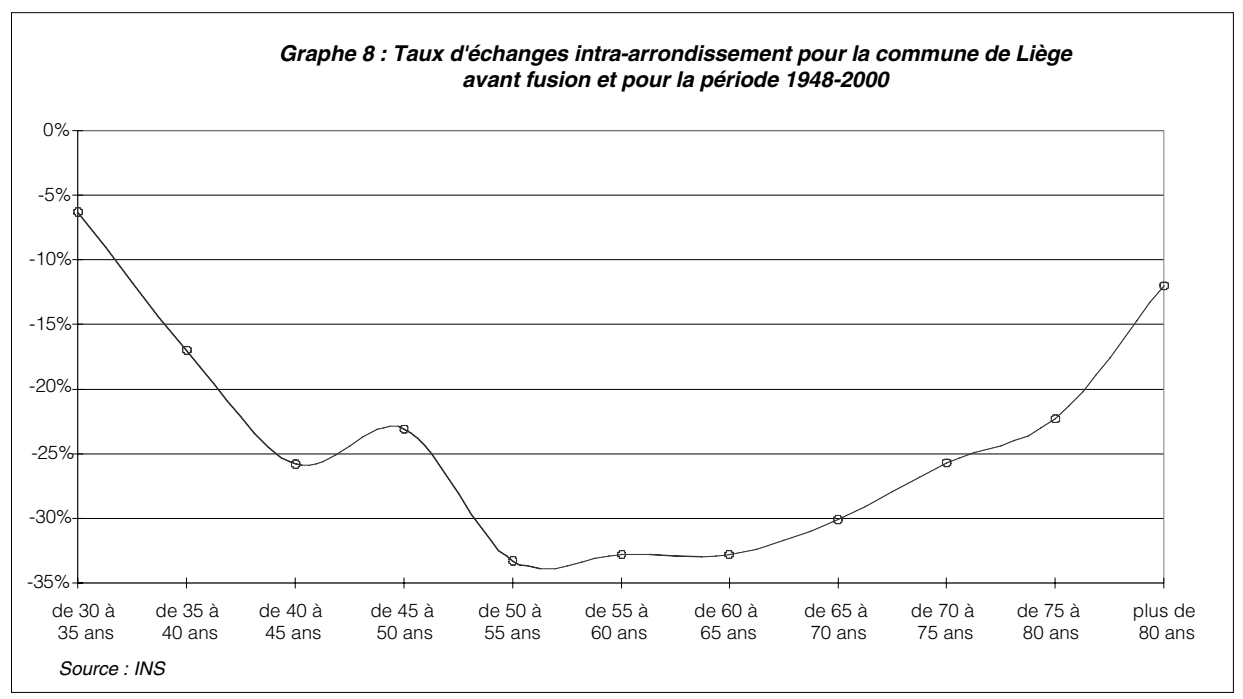

outre, la variable n'a plus été pondérée par la durée et la valeur reprise correspond au taux brut. Il ne s'agit donc plus d'un taux annuel ${ }^{11}$.

La temporalité longue des statistiques reprises dans le graphique 8 permet de mieux rendre compte de l'effet de génération, c'est-à-dire de comportements déterminés par l'appartenance à une cohorte née pendant une même période. À ce propos, on observe que les taux d'exurbanisation les plus importants à l'année 2000 sont le fait des groupes compris entre 50 et 65 ans. Pour ces cohortes ayant atteint la période d'élargissement du ménage lors du paroxysme périurbain des années 1970 (cohortes âgées de 20 à 35 ans en 1970), ce sont près de $35 \%$ des effectifs qui ont finalement abandonné la ville centrale ${ }^{12}$. Afin d'interpréter la plus faible exurbanisation du groupe compris entre 45 et 50 ans (taux de départ de $23 \%$ ), c'est sans conteste au contexte économique qu'il faut, de nouveau, faire référence. Ayant, pour leur part, atteint la période d'élargissement du ménage lors de la crise du début des années 1980 (cohortes âgées de 25 à 30 ans en 1980), les contraintes économiques de cette période les ont limité dans leur projet d'exode urbain. Avec le groupe quinquennal précédent des 40-45 ans en 2000 (20 à 25 ans en 1980), on retrouve une exurbanisation plus forte (taux de départ de $26 \%$ ), sans doute en grande partie effectuée lors de la seconde vague périurbaine de la fin des années 1980.

11. Mode de calcul de la variable présentée dans le graphique 8:

Taux d'échanges intra-arrondissement $=$ (Population réelle - Population attendue $) /$ Population attendue.

12. Signalons que cette valeur doit se comprendre comme un minimum. Elle est, en effet, basée sur l'hypothèse irréaliste d'une immigration nulle. 
Pour les classes d'âges ayant dépassé les 65 ans en 2000, nous observons le redressement de la courbe. À ce propos, il est utile de se remémorer que le solde migratoire de la ville centrale demeure négatif pour toutes ces catégories. En conséquence, un tel redressement ne peut s'expliquer que par des départs moins nombreux aux différentes classes d'âges. L'exurbanisation moins importante pour les cohortes de plus de 65 ans en 2000 indique que nous sommes toujours en présence de générations qui ne disposaient pas de la mobilité voiture lors de la période d'élargissement du ménage. Âgées de plus de 35 ans au début de la décennie 1970, elles étaient déjà installées avant que la croissance économique ne massifie la conduite automobile. Lors de la stabilisation familiale, leurs choix spatiaux étaient dès lors limités et, en 2000, on continue à les retrouver plus présents en ville centrale, dont ils représentent toujours plus de $20 \%$ de la population totale.

En France, la réalisation de grandes enquêtes statistiques a permis de vérifier que la conduite automobile se généralise au fil des générations. Dans ce pays, ce sont les "personnes qui se trouvent dans la deuxième partie de leur vie qui augmentent leurs mobilités, tandis que les croissances relatives les plus faibles sont le fait des jeunes adultes" (J.-P. Orfeuil, 1999, p. 11). Pour la Belgique, il n'existe aucune statistique disponible qui, de manière évolutive, aborde directement l'articulation entre l'âge et l'usage de l'automobile. Afin de partiellement combler cette lacune, nous avons traité les données sur les dépenses pour le secteur de l'automobile et notre approche a consisté dans la comparaison des deux enquêtes 1987-1988 et 2000 réalisées par l'INS sur les dépenses des ménages.

Au total, les dépenses liées à l'achat et à l'utilisation de l'automobile se sont accrues de $50 \%$ sur la période considérée (calcul hors inflation). Durant la même période, les dépenses pour les services de transports en commun ont régressé de $25 \%$. L'augmentation considérable des dépenses pour l'automobile doit être reliée à l'émergence d'une organisation urbaine dont le fonctionnement est de plus en plus dépendant de la conduite automobile, notamment en raison de l'étalement résidentiel.

Le traitement des deux enquêtes sur le budget des ménages conduit à vérifier l'hypothèse de la généralisation de l'usage de l'automobile au fil des générations. La croissance relative est, en effet, faible pour le groupe décennal des 20-29 ans (31\%) et élevée pour les personnes âgées de plus de 60 ans (89\%). Rappelons qu'entre 60 et 65 ans, on retrouve désormais les cohortes ayant initiés la périurbanisation massive du début des années 1970. Pour autant, comme nous venons de le constater, l'ensemble des cohortes ayant dépassé 65 ans en 2000 ne sont pas encore des habitués de la conduite automobile. D'ici les quinze prochaines années, il faut donc s'attendre à ce que la simple inertie du renouvellement des générations participe au gonflement des trafics automobiles. 


\section{LA DYNAMIQUE CENTRIFUGE DES FAMILLES EN FORMATION FACE AUX CONTRAINTES INDIVIDUELLES ET COLLECTIVES}

Il y a maintenant plusieurs décennies que la banalisation de la conduite dilate les territoires de la quotidienneté urbaine. En Belgique, comme d'ailleurs au sein d'autres contextes européens, il en résulte une profonde recomposition des villes. Les éléments rassemblés dans cet article indiquent que ce sont les ménages dont la vie familiale nécessite de l'espace qui ont initié cette transition urbaine, il y a de cela quatre décennies. C'est en effet à partir des années 1960 que les familles en formation se sont massivement dirigées vers les franges périurbaines. Alors que les contraintes de la mobilité quotidienne les avaient jusqu'alors maintenu en ville centrale ou en première couronne, la banalisation de la conduite automobile les a alors délivré de la concurrence pour l'espace urbain, en élargissant considérablement la zone potentielle d'installation de la "maisonnée".

Face à la dynamique centrifuge des familles en formation, la mise en perspective temporelle des relations entre cycle de vie et mobilité résidentielle a également conduit à identifier différentes forces centripètes. En Belgique, plutôt que les freins de la planification spatiale, ce sont essentiellement des contraintes à caractère socio-économiques qui ont conduit à limiter la dynamique périurbaine. Lors des dernières décades, c'est tout d'abord la crise économique du début de la décennie 1980 qui, un temps, a limité l'exurbanisation et la croissance des périphéries. Lors de la décennie 1990, c'est ici la précarité de l'emploi post-fordiste qui a conduit à intensifier les intra-urbanisations. En raison des difficiles stabilisations professionnelles et personnelles, les jeunes adultes flexibilisés demeurent, en effet, plus longtemps captifs de l'offre locative des tissus urbains centraux.

La mise en perspective temporelle des relations entre choix résidentiels et cycles de vie montre que le remplacement progressif des générations participe toujours à la dynamique centrifuge de la recomposition des villes. Au-delà de 65 ans en 2000, on observe, en effet, la "survivance" de choix résidentiels et de pratiques de mobilité antérieurs à la massification de l'automobile. Sur le strict plan des structures par âges, la transition urbaine s'achèvera lorsque, au fil du renouvellement des générations, cet effet de cohorte n'interférera plus avec l'effet du cycle de vie. Pour le contexte belge, une quinzaine d'années devra encore s'écouler avant que les différentes cohortes ne soient toutes formées d'individus habitués à la conduite et aux pratiques résidentielles qu'elle autorise.

En raison du relâchement des contraintes de mobilité, l'offre physiquement accessible n'est donc plus le principal facteur limitant pour les choix résidentiels des familles en formation. Aujourd'hui, c'est l'offre financièrement accessible qui constitue la première contrainte à l'établissement de la maisonnée, ce qui étale l'urbanisation, allonge inutilement les déplacements et augmente le budget que les ménages doivent consacrer à l'automobile. À l'instar de la situation décrite par V. Kaufmann et al. (2001), nous constatons donc le caractère contraignant du 
marché du logement et son incapacité à répondre de manière spatialement adaptée aux besoins des habitants, notamment pour les familles en formation.

Pour limiter les surcharges collectives et privées, il faut s'interroger sur les mécanismes de l'étalement et établir les modalités nécessaires à la production d'une offre résidentielle qui, bien que située dans la trame urbaine ou en première périphérie, puisse être adaptée aux besoins des familles contemporaines. Maximiser le desserrement en minimisant l'étalement impliquerait notamment que la collectivité intervienne sur le marché des terres constructibles afin de mettre en œuvre les abondants potentiels fonciers non valorisés des banlieues les plus proches. En parallèle, compléter l'intervention par une action sur l'étalement de pression foncière nécessiterait une modulation spatiale plus poussée des aides au logement, par exemple via des primes ou des prêts à taux avantageux conditionnés à une localisation centrale.

Répondre aux objectifs de la mobilité durable par la limitation des déplacements implique également de stopper la dépopulation urbaine. À travers la prise en compte des cycles de vie, il apparaît que le rôle des enfants est central dans l'exode urbain. L'idée communément admise est, en effet, qu'un milieu "campagnard" est la meilleure solution pour élever une famille. Limiter l'exurbanisation des familles et soutenir la mixité générationnelle de la trame urbaine nécessiteraient d'intervenir à la fois sur les équipements et sur la qualité des espaces publics. À ce propos, nous pouvons notamment souligner le besoin d'adapter la gestion de la mobilité urbaine à la vulnérabilité des enfants (Commission Européenne DGXI, 2002). Plus globalement, il est sans doute urgent de porter la réflexion sur le thème "les enfants dans la ville" afin de déterminer les conditions nécessaires à l'inversion de cette fuite devant l'urbain, cela notamment pour éviter l'étalement massif des actuelles familles juvéniles flexibles lorsqu'elles participeront à la natalité.

\section{BIBLIOGRAPHIE}

ASCHER François (1995). Métapolis ou l'avenir des villes. Paris : Odile Jacob.

ASCHER François (2000). Ces événements nous dépassent, feignons d'en être les organisateurs. Essai sur la société contemporaine. La Tour d'Aigues: Éd. de l'Aube, coll. Monde en cours.

BARTHE-BATSALLE H., BRÜCK L., DE KEERSMAECKER M.-L., GEORGES X., HALLEUX J.-M., LAMBOTTE J.-M., MARÉCHAL L. et ROUSSEAUX V. (2002). Les Coûts de la désurbanisation. Namur: Ministère de la Région wallonne, DGATLP, Études et Documents-CPDT 1.

BEAUCIRE F. (2001). "Sur la relation transports/urbanisme". Mobilité et territoire, Paris: Ministère de l'Équipement, des transports et du logement, Contributions au PREDIT, Direction de la Recherche et des Affaires Scientifiques et Techniques. 
BOURDIEU P. avec la coll. de BOUHEDJA S., CHRISTIN R. et GIVRY C. (1990). "Un placement de bon père de famille. La maison individuelle: spécificité du produit et logique du champ de production". Actes de la recherche en sciences sociales, $\mathrm{n}^{\circ} 81-82$, p. 6-33.

BRÜCK L., HALLEUX J.-M., JEHIN J.-B., LAMBOTTE J.-M., MAIRY N., VAN HOOF T. et SAVENBERG S., sous la direction de MÉRENNE-SCHOUMAKER B. et VAN HECKE E. (2000). Summary of the project "Residential Behaviour of Households and Sustainable Development Issue". Étude réalisée avec le soutien des SSTC dans le cadre du Plan d'Appui au Développement durable, SEGEFA-ISEG, Université de Liège, KULeuven, inédit.

COMMISSION EUROPÉENNE DGXI (2002). Villes d'enfants, Villes d'avenir. Direction Générale de l'environnement.

DE KEERSMAECKER M.-L. (2002). "Approche des coûts de la désurbanisation du point de vue de la cohésion sociale et territoriale». Les Coûts de la désurbanisation. Namur: Ministère de la Région wallonne, DGATLP, Études et Documents-CPDT 1, p. 103-121.

DE WASSEIGE Y., LAFFUT M., RUYTERS C. et SCHEILPER P. (2000). "Bassins d'emplois et régions fonctionnelles. Méthodologie et définitions des bassins d'emplois belges". Namur: Ministère de la Région wallonne, Service des Études et de la Statistique, Discussion Papers, $\mathrm{n}^{\circ} 2005$.

DUBOIS Olivier, GABRIEL Ingrid, HALLEUX Jean-Marie et MICHEL Quentin (2002). Révision des plans de secteur et mécanismes fonciers en Wallonie: objectifs politiques, outils juridiques et mise en ouvre. Namur: Ministère de la Région wallonne, DGATLP, coll. Études et Documents-CPDT 2.

DUBOIS-TAINE Geneviève et CHALAS Yves, dir. (1997). La Ville émergente. La Tour d'Aigues: Éd. de l'Aube, coll. Monde en cours.

DUPUY Gabriel (1995). L'Auto et la ville. Paris : Flammarion, coll. Dominos.

EGGERICK T. et CAPRON C. (2001). "Rurbanisation et périurbanisation dans le centre de la Wallonie: une approche socio-démographique». Espace, Populations, Société, n 1-2, p. 123-137.

FOUCHIER Vincent (1997). Les Densités urbaines et le développement durable. Le cas de l'Ile-de-France et des villes nouvelles. Paris: Édition du S.G.V.N., La Documentation française.

FOUCHIER Vincent (2001). "Mesurer l'étalement, la dédensification, le desserrement: différentes formes de gain d'espaces en Ile-de-France". La Ville aux champs, ADEF, p. 29-48.

GRIMMEAU J.-P., VAN CRIEKINGEN M. et ROELANDTS M. (1998). "Les migrations d'émancipation en Belgique". Espace, Populations, Société, nº 2, p. 235-247.

HALLEUX J.-M. (1999). "Dépopulation de la Ville de Liège et diffusion périurbaine: quels processus pour quelles pistes de solutions?". Wallonie, Revue $d u$ Conseil économique et social de la Région Wallonne, n 57, p. 44-53. 
HALLEUX J.-M. (2001). "Évolutions des organisations urbaines et mobilités quotidiennes: espace de référence et analyse des processus". L'Espace géographique, tome $30, \mathrm{n}^{\circ} 1$, p. 67-80.

HALLEUX J.-M. (2002). "Les forces centrifuges de la mobilité facilitée, le système des quotidiennetés urbaines et la recomposition des espaces urbains". Premier Colloque du Groupe de Travail Mobilités spatiales et fluidité sociale, AISLF, Louvain-la-Neuve, en cours de publication.

HALLEUX J.-M. (2002). "Les forces centrifuges de la mobilité facilitée, le système des quotidiennetés urbaines et la recomposition des espaces urbains". Mobilités, fluidités... libertés ? Bruxelles: Publication des Facultés universitaires Saint-Louis, p. 201-216

KAUFMANN Vincent, JEMELIN Christophe et GUIDEZ Jean-Marie (2001). Automobile et modes de vie urbain: quel degré de liberté? Paris: La Documentation française.

Ministère de la Région wallonne (2002). Évaluation des besoins des activités. Problématique de leur localisation. Premier volume: évaluation des besoins et des disponibilités foncières pour les activités résidentielles. Rapport final de la subvention 2001, ULB - UCL - ULg/FUSAGx, inédit.

NEWMAN P.W.G. et KENWORTHY J.-R. (1989). Cities and automobile dependance: an international sourcebook. Aldershot: Gower.

ORFEUIL J.-P. (1999). "Les évolutions majeures de la mobilité". Urbanisme, Horssérie $n^{\circ} 12$, p. 10-11.

PATTYN M. et VAN DER HAEGEN H. (1979). "Les régions urbaines belges". Études statistiques, ${ }^{\circ}$ 56, p. 3-18.

SAVENBERG S. et VAN HECKE E. (1998). Typologie van de migraties naar leeftijd. DWTC - Hefbomen voor een beleid gericht op duurzame ontwikkeling, Instituut voor Sociale en Economische Geografie, K.U.Leuven, inédit.

VAN CRIEKINGEN Mathieu. (2001). La Rénovation résidentielle à Montréal et à Bruxelles. Dynamiques, impacts sociaux et rôle des pouvoirs publics. Thèse présentée en vue de l'obtention du grade de docteur en Sciences géographiques, Laboratoire de Géographie humaine, ULB, inédit.

VAN DER HAEGEN H., VAN HECKE E. et JUCHTMANS G. (1996). "Les régions urbaines belges en 1991 ". Études statistiques, n 104.

VAN DER HAEGEN H., VAN HECKE E. et SAVENBERG S. (2000). "Belgians on the move: population distribution from a historical perspective". BELGEO, $\mathrm{n}^{\circ} 1$ 2-3-4, p. 173-188.

WIEL Marc (1999a). "Mobilité, interactions sociales et dynamiques territoriales". Urbanisme, Hors-série n 12 , p. 54-57.

WIEL Marc (1999b). La Transition urbaine ou le passage de la ville pédestre à la ville motorisée. Sprimont: P. Mardaga. 